\title{
Designing quadratic nonlinear photonic crystal fibers for soliton compression to few- cycle pulses
}

Bache, Morten; Moses, Jeffrey; Lægsgaard, Jesper; Bang, Ole; Wise, Frank

Published in:

European Conference on Lasers and Electro-Optics, 2007 and the International Quantum Electronics Conference. CLEOE-IQEC 2007.

Link to article, DOI:

10.1109/CLEOE-IQEC.2007.4386534

Publication date:

2007

Document Version

Publisher's PDF, also known as Version of record

Link back to DTU Orbit

Citation (APA):

Bache, M., Moses, J., Lægsgaard, J., Bang, O., \& Wise, F. (2007). Designing quadratic nonlinear photonic crystal fibers for soliton compression to few-cycle pulses. In European Conference on Lasers and Electro-Optics, 2007 and the International Quantum Electronics Conference. CLEOE-IQEC 2007. (pp. CJ1-3-WED). IEEE. https://doi.org/10.1109/CLEOE-IQEC.2007.4386534

\section{General rights}

Copyright and moral rights for the publications made accessible in the public portal are retained by the authors and/or other copyright owners and it is a condition of accessing publications that users recognise and abide by the legal requirements associated with these rights.

- Users may download and print one copy of any publication from the public portal for the purpose of private study or research.

- You may not further distribute the material or use it for any profit-making activity or commercial gain

- You may freely distribute the URL identifying the publication in the public portal 


\title{
Designing Quadratic Nonlinear Photonic Crystal Fibers for Soliton Compression to Few-cycle Pulses
}

\author{
Morten Bache ${ }^{1}$, Jeffrey Moses ${ }^{2}$, Jesper Lagsgaard ${ }^{1}$, Ole Bang ${ }^{1}$, Frank W. Wise ${ }^{2}$ \\ 1. COM•DTU, Technical University of Denmark, Bld. 345v, DK-2800 Lyngby, Denmark. \\ 2. Department of Applied and Engineering Physics, Cornell University, Ithaca, New York 14853.
}

Second-harmonic generation (SHG) in the limit of large phase mismatch, given by $\Delta \beta=\beta_{2}$-2 $\beta_{1}$, effectively induces a Kerr-like nonlinear phase shift on the fundamental wave (FW). The phase mismatch determines the sign and magnitude of the effective Kerr nonlinearity, making large negative phase shifts accessible. This self-defocusing nonlinearity can be used to compress a pulse when combined with normal dispersion, and problems normally encountered due to selffocusing in cubic media are avoided. Thus, having no power limit, in bulk media a self-defocusing soliton compressor can create high-energy near single-cycle fs pulses [1]. However, the group-velocity mismatch (GVM) between the FW and second harmonic (SH), given by the inverse group velocity difference $d_{12}=1 / v_{g, 1}-1 / v_{g, 2}$, limits the pulse quality and compression ratio. Especially very short input pulses ( $<100 \mathrm{fs})$ experience a Raman-like effect with a characteristic time $T_{\mathrm{R}, \mathrm{SHG}}=2 \mid d_{12} / / \Delta \beta[1 \mathrm{c}]$. Here we address the limits imposed by GVM by using thermally-poled silica photonic crystal fibers (PCFs) for cascaded quadratic $\left(\chi^{(2)}: \chi^{(2)}\right)$ soliton compression. In standard silica fibers strong effective quadratic nonlinearities around $1 \mathrm{pm} / \mathrm{V}$ have been created with poling. PCFs are instead interesting because they have very strong wave-guide dispersion that can be tailored: for SHG index-guiding silica PCFs with a triangular hole-arrangement can have zero GVM for any FW wavelength $\lambda_{1}>780 \mathrm{~nm}$ by adjusting the PCF hole pitch $A$ and hole diameter $d$ [2]. Our simulations predict that high-quality compression to few-cycle pulses in poled PCFs is possible. Such a waveguided geometry can extend the compression technique to lower-energy pulses and produce uniformly compressed beams.

The system is modeled by generalized coupled nonlinear Schrödinger equations with quadratic nonlinear terms, selfsteepening and Raman effects. The rescaled equations have the usual fiber optics Kerr soliton number $N_{\text {Kerr }}$ as well as a new SHG one $N_{\mathrm{SHG}}$. We found that FW soliton compression requires $N_{\mathrm{SHG}}>N_{\mathrm{Kerr}}$. This poses an upper limit on $\Delta \beta$ as

$$
\bar{N}^{2}=\frac{N_{\mathrm{SHG}}^{2}}{N_{\mathrm{Kerr}}^{2}}>1 \Rightarrow|\Delta \beta|<\Delta \beta_{c}=\frac{\omega_{1} d_{\mathrm{ef}}^{2} A_{\mathrm{effr}}^{\mathrm{Kerr}}}{c n_{\mathrm{eff}, 1} n_{\mathrm{eff}, 2} n_{2} A_{\mathrm{eff}}^{\mathrm{SHG}}}, \quad N_{\mathrm{Kerr}}^{2}=L_{\mathrm{D}, 1} \frac{P_{1, \mathrm{in}} 2 \omega_{1} n_{2}}{\varepsilon_{0} c^{2} n_{\mathrm{eff}, 1} A_{\mathrm{effr}, 1}^{\mathrm{Ker}}}, \quad N_{\mathrm{SHG}}^{2}=L_{\mathrm{D}, 1} \frac{P_{1, \mathrm{in}} 2 \omega_{1}^{2} d_{\mathrm{eff}}^{2}}{\varepsilon_{0} c^{3} n_{\mathrm{eff}, 1}^{2} n_{\mathrm{eff}, 2}|\Delta \beta| A_{\mathrm{eff}}^{\mathrm{SHG}}}
$$

$L_{\mathrm{D}, 1}$ : FW dispersion length; $P_{1, \text { in }}$ FW input power; $n_{2}$ : Kerr nonlinear refractive index; $d_{\mathrm{eff}}$ : effective quadratic nonlinearity; $n_{\mathrm{eff}, j}$ : effective PCF mode indices; $A_{\mathrm{eff}}^{\mathrm{SHG}}$ : SHG effective mode area; $A_{\mathrm{eff}, 1}^{\mathrm{Kerr}}$ : FW cubic effective mode area. GVM poses a lower compression limit: $\Delta \beta>\Delta \beta_{\mathrm{sr}}=4 \pi\left|d_{12}\right| / T_{1 \text {,in, }}$, with $T_{1 \text {,in }}$ the FW input pulse length. Only in this stationary regime is the nonlinear phase shift built up before GVM separates the fields [1]. Together these limits define a $\Delta \beta$-"window" of possible compression. The window opens $\left(\Delta \beta_{c}>\Delta \beta>\Delta \beta_{\mathrm{sr}}\right)$ when GVM is small and $d_{\mathrm{eff}}^{2} / n_{2}$ is large.

Based on Ref. [2], Table 1 shows selected PCF designs with medium, small and zero GVM. We had to choose a low pitch $A$ to have both reduced GVM and normal dispersion. The GVM reduction was unfortunately accompanied by large phase mismatches $>100 \pi / \mathrm{mm}$. Thus, achieving $\Delta \beta_{c}>\Delta \beta$ would require unrealistically large $d_{\text {eff-values }}(3-5 \mathrm{pm} / \mathrm{V})$. With a more realistic $d_{\mathrm{eff}}=1 \mathrm{pm} / \mathrm{V}, \Delta \beta_{c}<\Delta \beta$, so no compression would occur. We solve this issue by using quasi-phase matching (QPM), where a grating on the quadratic nonlinearity with pitch $\Lambda_{\mathrm{QPM}}$ gives an effective phase mismatch $\Delta \beta_{\text {eff }}=\Delta \beta-2 \pi / \Lambda_{\mathrm{QPM}}$. $\Lambda_{\mathrm{QPM}}$ is then adjusted so $\Delta \beta_{\text {eff }}<\Delta \beta_{c}$, where $\Delta \beta_{c}$ is found by taking into account that QPM effectively reduces $d_{\text {eff }}$ by $2 / \pi$. To stay in the cascading limit $\left(\Delta \beta_{\text {eff }}>0\right)$, a slightly "mismatched" grating is used $\Lambda_{\mathrm{QPM}} \neq 2 \pi /|\Delta \beta|$.

Table 1. Selected poled silica PCF designs for $\lambda_{1}=1060 \mathrm{~nm}, \Lambda=0.9 \mu \mathrm{m}, d_{\mathrm{eff}}=1 \mathrm{pm} / \mathrm{V}$ and a $400 \mathrm{fs}$ FWHM input pulse.

\begin{tabular}{|c||c|c|c|c|c|c|c|c|c|c|c|c|c|}
\hline & $\begin{array}{c}d \\
\mu \mathrm{m}\end{array}$ & $\begin{array}{c}\Delta \beta \\
\mathrm{mm}^{-1}\end{array}$ & $\begin{array}{c}d_{12} \\
\mathrm{fs} / \mathrm{mm}\end{array}$ & $\begin{array}{c}\mathrm{FW} \mathrm{GVD} \\
\mathrm{fs}^{2} / \mathrm{mm}\end{array}$ & $\begin{array}{c}A_{\mathrm{eff}}^{\mathrm{SHG}} \\
\mu \mathrm{m}^{2}\end{array}$ & $\begin{array}{c}A_{\text {eff, }}^{\mathrm{K}} \\
\mu \mathrm{m}^{2}\end{array}$ & $\begin{array}{c}\Delta \beta_{c} \\
\mathrm{~mm}^{-1}\end{array}$ & $\begin{array}{c}\Delta \beta_{\mathrm{sr}} \\
\mathrm{mm}^{-1}\end{array}$ & $\begin{array}{c}A_{\mathrm{QPM}} \\
\mu \mathrm{m}\end{array}$ & $\begin{array}{c}\Delta \beta_{\text {eff }} \\
\mathrm{mm}^{-1}\end{array}$ & $\begin{array}{c}T_{R, S H G, \text { eff }} \\
\mathrm{fs}\end{array}$ & $\begin{array}{c}P_{1, \text { in }} \\
\mathrm{kW}\end{array}$ & $\begin{array}{c}P_{1, \text { out }} \\
\mathrm{kW}\end{array}$ \\
\hline \hline 1 & 0.63 & 721.29 & 0 & 41 & 1.26 & 1.22 & 30.2 & 0 & 8.83 & 9.71 & 0.0 & 0.5 & 3.6 \\
\hline 2 & 0.54 & 596.30 & -33 & 87 & 1.60 & 1.56 & 29.6 & 1.81 & 10.71 & 9.63 & 6.8 & 1.3 & 10.6 \\
\hline 3 & 0.45 & 473.56 & -72 & 138 & 2.34 & 2.29 & 29.1 & 3.91 & 13.54 & 9.52 & 14.8 & 3.0 & 7.2 \\
\hline
\end{tabular}

We performed numerics choosing $\Lambda_{\mathrm{QPM}}$ to have $\bar{N}=1.76$ and $P_{1, \text { in }}$ to have $N_{\mathrm{SHG}}=14.5$. Case 1 and 2 from Table 1 gave good-quality compression from $400 \mathrm{fs}$ to $\sim 14 \mathrm{fs}$, while case 3 with medium GVM gave a $\sim 37 \mathrm{fs}$ asymmetric pulse. It is therefore very important to reduce GVM because of a reduced characteristic time of the Raman-like perturbation $T_{R, \mathrm{SHG}, \text { eff }}=2\left|d_{12}\right| / \Delta \beta_{\text {eff. }}$. In fact case 3 has a $T_{\mathrm{R}, \mathrm{SHG} \text {,eff }}$-value which is 5 times larger than the material Raman-time of silica $\left(T_{\mathrm{R}} \sim 3 \mathrm{fs}\right)$. Additional advantages of small GVM are a reduced lower limit $\Delta \beta_{\mathrm{sr}}$, which for short $<100$ fs input pulses becomes important, as well as lower power/energy needed to compress the pulse. In fact strong GVM leads to large FW depletion, thereby reducing the energy in the compressed pulse. Finally, we observed the best pulse quality not with zero GVM, case 1, but rather with a small GVM, case 2, because GVM removes some detrimental oscillations in the pulse center. The output power levels imply that efficient, high-intensity compression of nJ pulses is possible.

\section{References}

1. (a) X. Liu, L. Qian, and F. W. Wise, Opt. Lett. 24, 1777-1779 (1999); (b) S. Ashihara et al., J. Opt. Soc. Am. B 19, 2505-2510 (2002); (c) J. Moses and F. W. Wise, Opt. Lett. 31, 1881-1883 (2006).

2. M. Bache, H. Nielsen, J. Lægsgaard and O. Bang, Opt. Lett. 31, 1612-1614, (2006). 